\title{
In Vitro Evaluation of Radio-Labeled Aerosol Delivery Via a Variable-Flow Infant CPAP System
}

\author{
Kimberly D Farney RRT, Brandon T Kuehne RRT MBA, Laurie A Gibson RT (N), \\ Leif D Nelin MD, and Edward G Shepherd MD
}

\begin{abstract}
BACKGROUND: Nasal CPAP is widely used in neonatal ICUs. Aerosolized medications such as inhaled steroids and $\boldsymbol{\beta}$ agonists are commonly administered in-line through nasal CPAP, especially to infants with bronchopulmonary dysplasia. We hypothesized that aerosol delivery to the lungs via variable-flow nasal CPAP in an in vitro model would be unreliable, and that the delivery would depend on the position of the aerosol generator within the nasal CPAP circuit. METHODS: We used a system that employed a test lung placed in a plastic jar and subjected to negative pressure. Simulated inspiration effort was measured with a heated-wire anemometer. We used technetium99m-labeled diethylene triamine penta-acetic acid as our aerosol. The nebulizer was placed either close to the humidifier or close to the nasal prongs in the circuit, and patient effort was simulated with a minute ventilation of $0.4 \mathrm{~L} / \mathrm{min}$. RESULTS: Relative aerosol delivery to the infant test lung with the nebulizer close to the humidifier was extremely low $(0.3 \pm 0.4 \%)$, whereas placing the nebulizer close to the nasal prongs resulted in significantly $(P<.001)$ improved delivery $(21 \pm 11 \%)$. Major areas of aerosol deposition with the nebulizer close to the humidifier versus close to the nasal prongs were: nebulizer $(10 \pm 4 \%$ vs $33 \pm 13 \%, P<.001)$, exhalation limb $(9 \pm 17 \%$ vs $26 \pm 30 \%$, $P=.23)$, and generator tubing $(21 \pm 11 \%$ vs $19 \pm 20 \%, P=.86)$. Placing the nebulizer close to the humidifier resulted in $59 \pm \mathbf{8 \%}$ of the aerosol being deposited in the inhalation tubing along the heater wire. CONCLUSIONS: Isotope delivery from an aerosol generator placed near the humidifier on variable-flow nasal CPAP was negligible in this in vitro setup; however, such delivery was significantly improved by locating the aerosol generator closer to the nasal CPAP interface. Key words: bronchopulmonary dysplasia; neonatal respiratory distress; inhalational administration; respiratory therapy; neonate. [Respir Care 2014;59(3):340-344. () 2014 Daedalus Enterprises]
\end{abstract}

\section{Introduction}

Bronchopulmonary dysplasia is the most common pulmonary complication of prematurity, and is associated with substantial neurodevelopmental risks. ${ }^{1-3}$ Numerous interventions have been proposed to treat bronchopulmonary dysplasia, including the routine use of nasal CPAP and inhaled steroids; however, data regarding their efficacy are

\footnotetext{
Ms Farney, Mr Kuehne, and Ms Gibson are affiliated with Respiratory Therapy, Neonatal Service Line; Dr Nelin is affiliated with the Center for Perinatal Research; and Dr Shepherd is affiliated with the Department of Neonatology, Nationwide Children's Hospital, Columbus, Ohio.

Ms Farney presented a version of this paper at the 56th AARC Congress, held December 6-9, 2010, in Las Vegas, Nevada.
}

The authors have disclosed no conflicts of interest. limited. ${ }^{4,5}$ Many patients with bronchopulmonary dysplasia receive aerosolized $\beta$ agonist therapy, although there is little data on which to base dosing, medication deposition,

See the Related Editorial on Page 443

or clinical response. ${ }^{5,6}$ It is not clear if the limited evidence of effectiveness of these interventions is due to intrinsic

Correspondence: Edward G Shepherd MD, Department of Neonatology, Nationwide Children's Hospital, 700 Children's Drive, Columbus OH 43205. E-mail: edward.shepherd@ nationwidechildrens.org.

DOI: $10.4187 /$ respcare.01904 
factors or to difficulties achieving measurable deposition in the target tissues, especially for infants on nasal CPAP.

The Aeroneb Solo (Aerogen, Galway, Ireland) disposable nebulizer is commonly used in the hospital setting. Studies suggest this generator may be superior to some others at delivering medication to the lung in mechanically ventilated patients. ${ }^{7}$ However, little is known about how much aerosol is delivered to the patient's lung when the Aeroneb Solo is used in-line with a variable-flow infant nasal CPAP system. In addition, nebulizer placement within the nasal CPAP circuit is limited to either the humidifying chamber or the heater wire-nasal CPAP generator interface. We hypothesized that placing the Aeroneb Solo nebulizer closer to the nasal interface (ie, further from the humidifier) would increase test lung deposition of aerosolized isotope. We tested the Aeroneb Solo with an infant variable-flow nasal CPAP circuit and a lung model. We measured isotope deposition using scintigraphy.

\section{Methods}

The study circuit consisted of a heated-wire circuit (RT324, Cardinal Health, Dublin, Ohio), a nasal CPAP generator (Infant Flow SiPAP, CareFusion, San Diego, California), the Aeroneb Solo nebulizer (a new one for each test run), and an institutionally constructed infant test-lung system. The test-lung was subjected to negative pressure by means of a timed valve mechanism connected to a vacuum system. Simulated inspiration effort was triggered by increasing the negative pressure surrounding the test lung, and was measured with a heated-wire anemometer (CareFusion, San Diego, California), the flow signal from which was integrated into a volume measurement by a ventilator (Avea, CareFusion, San Diego, California).

We chose to study only the variable-flow nasal CPAP device because it reduces the work of breathing in infants and thus is the primary modality within our unit. ${ }^{8,9}$ We use variable-flow nasal CPAP for all near-term and post-term infants, who comprise those most likely to be prescribed aerosolized respiratory medications in our unit. We tested the device solely in CPAP mode because that is our standard practice.

We set the nasal CPAP for a base flow of about $8 \mathrm{~L} / \mathrm{min}$, to achieve a CPAP of $6 \mathrm{~cm} \mathrm{H}_{2} \mathrm{O}$, with no system leak. The test-lung was set at a tidal volume of $45 \mathrm{~mL}$ and a minute ventilation of $0.4 \mathrm{~L} / \mathrm{min}$. Depending on the size of the infant, this represents a tidal volume of $10-20 \mathrm{~mL} / \mathrm{kg} /$ breath. While that tidal volume range is larger than typical tidal volumes in healthy preterm newborns, we believed that these settings would maximize isotope deposition in the artificial lung, and thereby minimize the possibility of an artifactual decrease in lung deposition of the isotope. We nebulized $3 \mathrm{~mL}$ of saline with technetium-99m-labeled diethylene triamine penta-acetic acid (DTPA), de-

\section{QUICK LOOK}

\section{Current knowledge}

Nasal CPAP is commonly used in neonatal intensive care, and is often coupled with delivery of aerosolized medications. The optimal placement of the nebulizer in the CPAP circuit to maximize medication delivery is unknown.

\section{What this paper contributes to our knowledge}

In an in vitro model of infant nasal CPAP, radio-labeled aerosol delivery to a test lung was substantially improved by moving the nebulizer closer to the patient.

livered over $15 \mathrm{~min}$. We conducted the tests with the nebulizer placed at the humidifying chamber (Fisher \& Paykel, Auckland, New Zealand) (position A), and placed at $32 \mathrm{~cm}$ from the nasal prongs (position B) (Fig. 1). Position B (at the junction of the heated-wire circuit and the nasal CPAP generator) was the closest practical placement of the nebulizer to the nasal prongs, given the design of the circuit, and no commercially available devices exist to place a nebulizer within the nasal CPAP generator itself. With a gamma camera (Infinia Hawkeye, GE Healthcare, Little Chalfont, Buckinghamshire, United Kingdom) we imaged all the circuits prior to the delivering any aerosol, to determine the zero-exposure baseline (Fig. 2).

A total of 15 measurements were obtained. There were 6 measurements taken with the nebulizer placed in position $\mathrm{A}$, and 9 measurements were taken with the nebulizer placed in position B. After the isotope was delivered, the circuit was disassembled into 5 sections (nebulizer, heated-wire segment, patient segment, exhalation segment, and generator tubing) and analyzed for isotope deposition with the gamma camera and nuclear image analysis software (Xeleris, GE Healthcare, Little Chalfont, Buckinghamshire, United Kingdom, Fig. 3), with which we analyzed the deposition in the 5 circuit components.

Data are expressed as mean $\pm \mathrm{SD}$. Nebulizer position A was compared to position B with one-way analysis of variance, in statistics software (SigmaStat, Jandel Scientific, Carlsbad, California). Differences were considered statistically significance when $P<.05$.

\section{Results}

Placing the nebulizer at the humidifying chamber (position $\mathrm{A}$ ) resulted in a deposition of $<1 \%$ in the test lung, whereas placing the nebulizer $32 \mathrm{~cm}$ upstream from the nasal prongs (position B) resulted in substantial deposition of the isotope in the test lung system (Fig. 4). Indeed, with 


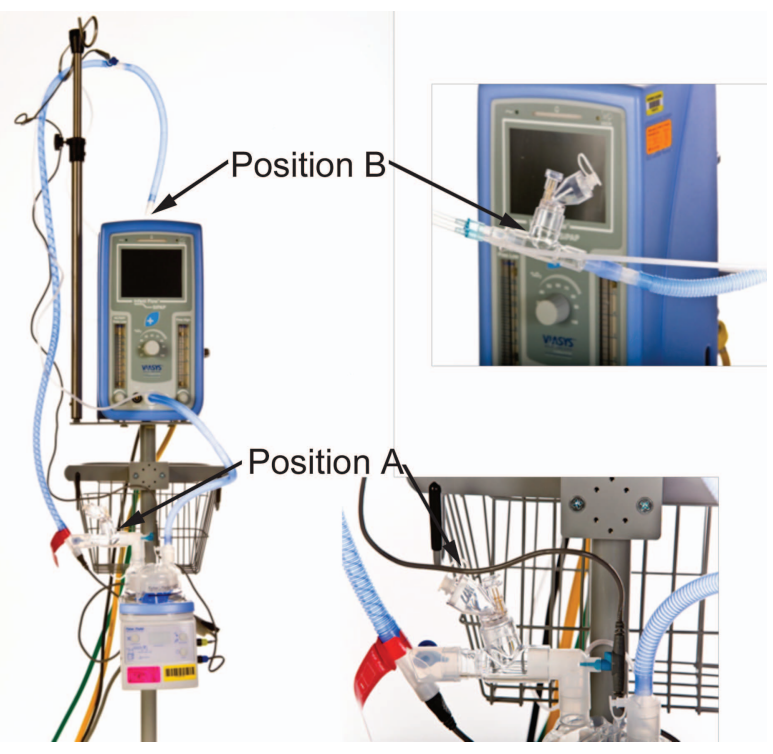

Fig. 1. Positions of the nebulizer in the variable-flow nasal CPAP circuit. Position A is near the humidifier. Position B is $32 \mathrm{~cm}$ upstream of the nasal prongs.

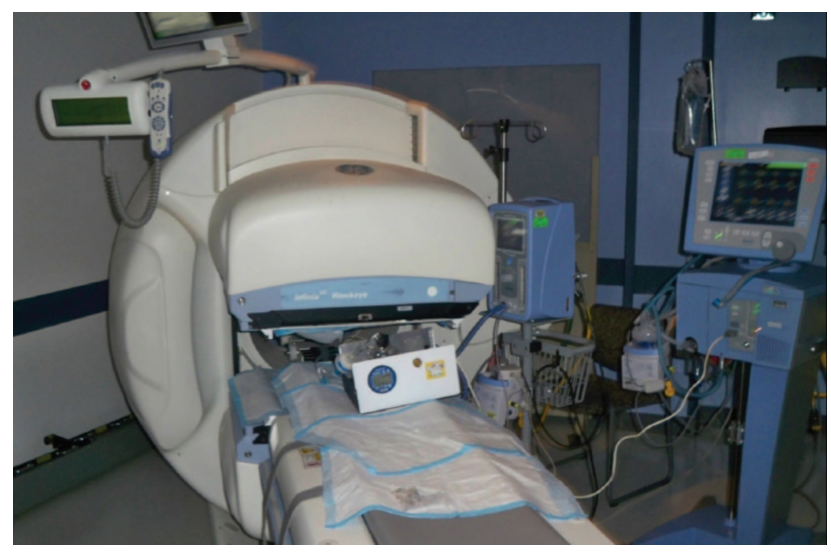

Fig. 2. Gamma camera, infant lung simulator, nasal CPAP circuit, nasal CPAP generator, and ventilator.

position A the vast majority of deposition was in the nebulizer and the heated-wire segment on the inhalation side of the circuit, and very little aerosol reached the patient or the exhalation circuit (see Fig. 3). Position B substantially increased deposition in the model lung and exhalation circuit (see Fig. 3). In addition, significantly more of the tracer remained in the nebulizer with position B than with position A (see Fig. 4).

\section{Discussion}

Aerosol deposition in the test lung was negligible when the nebulizer was placed at the humidifier (position A); the majority of tracer deposited in the heated-wire segment. Delivery to the test lung was markedly improved with nebulizer

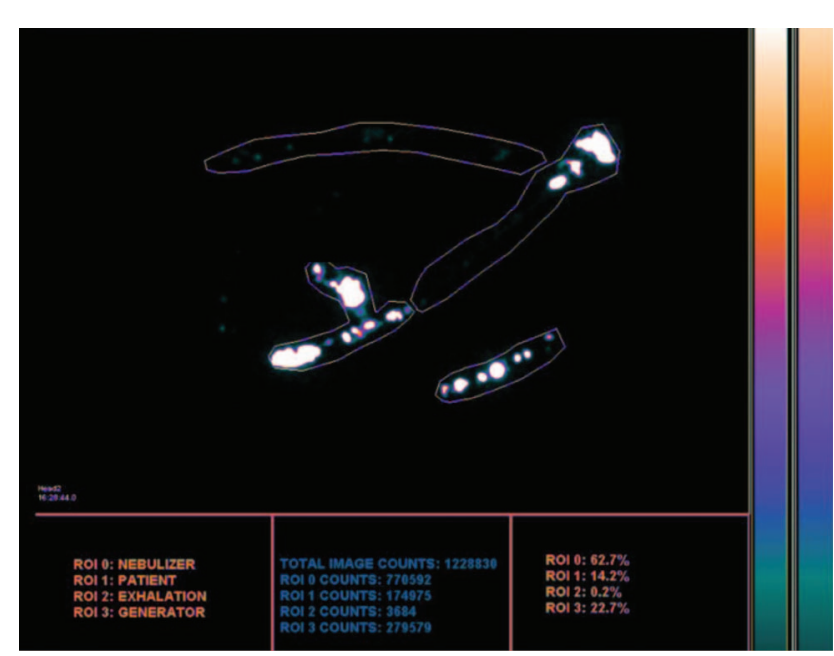

Fig. 3. Typical gamma camera image analysis shows the deposition of radio-labeled aerosol in the regions of interest (ROI). This image is from a test with the nebulizer placed $32 \mathrm{~cm}$ from the nasal prongs (position B).

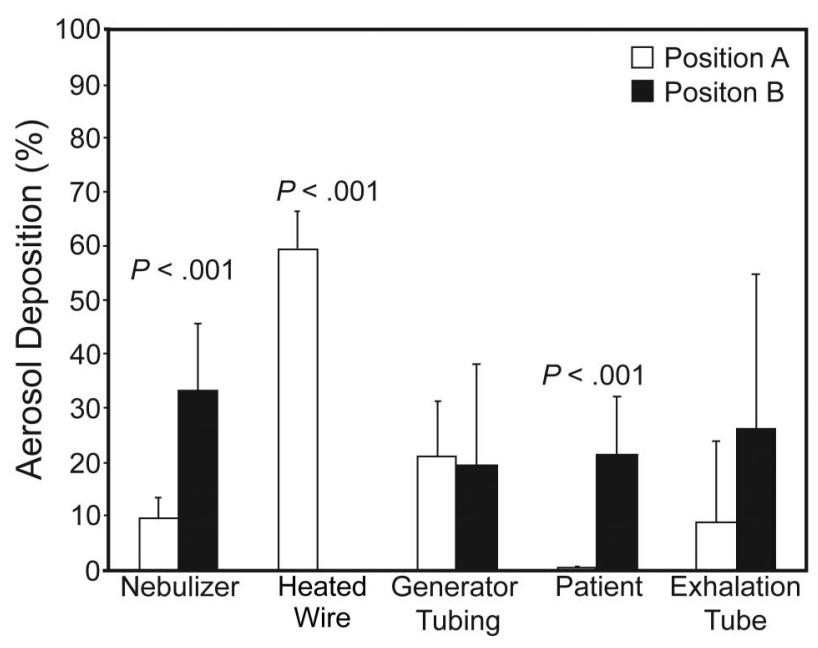

Fig. 4. Aerosol deposition in the 5 components of the test setup.

position $\mathrm{B}$, with concomitant reduction in tracer deposition elsewhere in the circuit. Together, these findings suggest that simply placing the nebulizer closer to the patient in the variable-flow nasal CPAP circuit improves aerosol delivery.

To the best of our knowledge, this is the first study to examine the delivery of radio-labeled aerosol to a test lung via a standard, variable-flow infant nasal CPAP interface. Position A was chosen to reflect the circuit set-up used for aerosol administration in our neonatal ICU, and we found very low lung deposition with position A. This is consistent with in vivo and in vitro reports that lung deposition is often very low. For example, O'Riordan et al ${ }^{10}$ found in adult patients ventilated via tracheostomy that $\sim 15 \%$ of a jet-nebulized charge was deposited in the lungs. In ventilated infants with bronchopulmonary dysplasia, Fok et al ${ }^{11}$ 
found that only about $2 \%$ of a jet-nebulized dose was deposited in the lung. In our test setup lung deposition was significantly improved with the nebulizer placed much closer to the nasal prongs. This finding on the effect of nebulizer location closer to the patient in the nasal CPAP circuit is consistent with other in vitro studies with adult ventilators and circuits. For example, Ari et al ${ }^{12}$ found that moving an ultrasonic nebulizer from a distal (close to the ventilator) to a proximal (close to the patient) position increased deposition in the test lung from $\sim 5 \%$ to $\sim 17 \%$. With a ventilator designed for noninvasive ventilation, pressures of $10 / 5 \mathrm{~cm} \mathrm{H}_{2} \mathrm{O}$, and a breathing frequency of 20 breaths/min, when a jet nebulizer was placed close to the ventilator the delivered aerosol was $\sim 9 \%$, and when placed close to the test lung the deposition increased to $\sim 16 \% .^{13}$ Thus, although lung deposition of aerosol is low in both adult and infant models, it appears that moving the nebulizer closer to the patient interface substantially improves aerosol delivery.

Although we report that isotope delivery in an in vitro model can be improved when using a variable-flow infant nasal CPAP circuit, the evidence for efficacy of aerosolized medications for the prevention and treatment of neonatal lung diseases is mixed. Inhaled steroids, for instance, may improve the rate of successful extubation in premature infants, but do not seem to affect the rate of bronchopulmonary dysplasia. ${ }^{5}$ Inhaled bronchodilators improve airway resistance but do not reduce the incidence of bronchopulmonary dysplasia.5,14 These disappointing clinical results may reflect either an intrinsic lack of efficacy of these medications or suboptimal delivery. Thus, we suggest that in future neonatal aerosol studies, particularly studies on nasal CPAP, documentation of drug delivery should be included in the study design.

\section{Limitations}

First, our in vitro results may not accurately reflect in vivo lung deposition, given the added complexities of the nasopharynx and airways. Second, the tidal volumes we chose were designed to maximize the aerosol delivery to the test lung and were therefore larger than typical prematureinfant tidal volumes. This could limit the clinical applicability of these findings in infants with smaller tidal volumes. Specifically, these findings may be generalized only to the relatively limited population of neonatal ICU patients who have similar tidal volumes and minute ventilation. Further, more premature infants with smaller tidal volumes still require substantial bias flow to maintain nasal CPAP, resulting in a greater ratio of bias flow to minute ventilation. It is possible, and perhaps even likely, that tissue deposition will be negatively affected by increasing the ratio of bias flow to minute ventilation. ${ }^{15}$ While our study and the study by Ari et al similarly suggest that aerosol delivery is decreased by increasing bias flow, our data do not confirm their findings with regard to nebulizer placement in the ventilator circuit. Specifically, Ari et al found that proximal (near the ventilator) versus distal (near the patient) placement of a vibrating mesh nebulizer was associated with substantially increased delivery of albuterol in a simulated adult model of mechanical ventilation, and increased delivery of albuterol at higher but not lower bias flow in a simulated pediatric model of mechanical ventilation. They hypothesized that these improvements were likely due to a "reservoir" effect within the inspiratory limb of the ventilator circuit. ${ }^{15} \mathrm{We}$ believe the differences between the studies are largely due to inherent differences in our models. Specifically, the bias flow necessary to maintain nasal CPAP is typically much higher (8 vs 2 or $5 \mathrm{~L} / \mathrm{min}$ ), and our tidal volumes were much smaller (45 vs $100 \mathrm{~mL}$ ). These differences in our model would likely minimize any potential reservoir effect of the inspiratory limb. Furthermore, deposition of isotope in this model may not reflect deposition of actual medication in a patient, as we were unable to test tagged medications. Finally, we did not measure aerosol particle size, which is an important determinant of aerosol delivery. Thus, we believe that our findings, though potentially clinically relevant, must be confirmed with additional in vitro and in vivo studies.

\section{Conclusions}

In our in vitro model of infant variable-flow nasal CPAP, lung aerosol deposition was substantially improved by moving the nebulizer closer to the patient.

\section{REFERENCES}

1. Gray PH, O’Callaghan MJ, Rogers YM. Psychoeducational outcome at school age of preterm infants with bronchopulmonary dysplasia. J Paediatr Child Health 2004;40(3):114-120.

2. Jeng SF, Hsu CH, Tsao PN, Chou HC, Lee WT, Kao HA, et al. Bronchopulmonary dysplasia predicts adverse developmental and clinical outcomes in very-low-birthweight infants. Dev Med Child Neurol 2008;50(1):51-57.

3. Kinsella JP, Greenough A, Abman SH. Bronchopulmonary dysplasia. Lancet 2006;367(9520):1421-1431.

4. Thomson MA. Continuous positive airway pressure and surfactant; combined data from animal experiments and clinical trials. Biol Neonate 2002;81(Suppl 1):16-19.

5. Pantalitschka T, Poets CF. Inhaled drugs for the prevention and treatment of bronchopulmonary dysplasia. Pediatr Pulmonol 2006; 41(8):703-708.

6. Shah SS, Ohlsson A, Halliday H, Shah vs Inhaled versus systemic corticosteroids for the treatment of chronic lung disease in ventilated very low birth weight preterm infants. Cochrane Database Syst Rev 2007;(4):CD002057.

7. Dubus JC, Vecellio L, De Monte M, Fink JB, Grimbert D, Montharu $\mathrm{J}$, et al. Aerosol deposition in neonatal ventilation. Pediatr Res 2005; 58(1):10-14. 
8. Courtney SE, Pyon KH, Saslow JG, Arnold GK, Pandit PB, Habib RH. Lung recruitment and breathing pattern during variable versus continuous flow nasal continuous positive airway pressure in premature infants: an evaluation of three devices. Pediatrics 2001;107(2):304-308.

9. Liptsen E, Aghai ZH, Pyon KH, Saslow JG, Nakhla T, Long J, et al. Work of breathing during nasal continuous positive airway pressure in preterm infants: a comparison of bubble vs variable-flow devices. J Perinatol 2005;25(7):453-458.

10. O'Riordan TG, Palmer LB, Smaldone GC. Aerosol deposition in mechanically ventilated patients: optimizing nebulizer delivery. Am J Respir Crit Care Med 1994;149(1):214-219.

11. Fok TF, Monkman S, Dolovich M, Gray S, Coates G, Paes B, et al. Efficiency of aerosol medication delivery from a metered dose inhaler versus jet nebulizer in infants with bronchopulmonary dysplasia. Pediatr Pulmonol 1996;21(5):301-309.
12. Ari A, Areabi H, Fink JB. Evaluation of aerosol generator devices at 3 locations in humidified and non-humidified circuits during adult mechanical ventilation. Respir Care 2010;55(7):837-844

13. Chatmongkolchart S, Schettino GP, Dillman C, Kacmarek RM, Hess DR. In vitro evaluation of aerosol bronchodilator delivery during noninvasive positive pressure ventilation: effect of ventilator settings and nebulizer position. Crit Care Med 2002;30(11):2515-2519.

14. Denjean A, Paris-Llado J, Zupan V, Debillon T, Kieffer F, Magny $\mathrm{JF}$, et al. Inhaled salbutamol and beclomethasone for preventing broncho-pulmonary dysplasia: a randomised double-blind study. Eur J Pediatr 1998;157(11):926-931.

15. Ari A, Atalay OT, Harwood R, Sheard MM, Aljamhan EA, Fink JB. Influence of nebulizer type, position, and bias flow on aerosol drug delivery in simulated pediatric and adult lung models during mechanical ventilation. Respir Care 2010;55(7):845-851.

This article is approved for Continuing Respiratory Care Education credit. For information and to obtain your CRCE

(free to AARC members) visit www.rcjournal.com 